\title{
Optimised analysis of community medical APP user experience under cognitive load theory
}

\author{
Peng $\mathrm{Han}^{1}$, Liyuan $\mathrm{Xu}^{2^{*}}$ and Xiaochen $\mathrm{Lv}^{1}$ \\ ${ }^{1}$ Qingdao HuanghaiUniversity, Qingdao City, Shandong Province,266555, China \\ ${ }^{2}$ Beijing Sport University, Beijing City, 100084, China
}

\begin{abstract}
Based on community medical APP as the carrier, this paper studies the emotional and experiential process of community doctors using mobile community medical APP under different cognitive loads, and puts forward the improvement of product optimization. Firstly, based on the cognitive load theory, objective user data is obtained through cognitive load control experiments on target users. Second, ask the target user to fill in the Positive and Negative Affect Scale and System Usability Scale to obtain subjective user data. Finally, analyze the user's subjective and objective data. Based on the analysis of experimental results and user observation, the improvement strategy is proposed to reduce the barriers to community doctors learning and using community medical apps.
\end{abstract}

\section{Introduction}

A good Internet medical model not only improves the efficiency of work, but also helps community doctors improve their clinical medical technology[1]. However, from the overall structure of community doctors in China, the level of cultural education of community doctors is relatively low. There are great differences in the application of Internet medical care. Therefore, how to improve the self-study ability of community doctors in the community medical system has become the focus of community Internet medical research content.Based on cognitive load theory, this study explores the operating experience of community doctors for community mobile medical system under different cognitive load conditions through cognitive load control experiments, Positive and Negative Affect Scale, System Usability Scale and so on. And combined with the experimental results, the optimization method of improving the community mobile diagnosis and treatment system is put forward.

\section{$2 \quad$ Literature Review}

\subsection{Cognitive load}

Cognitive load is done by Australian psychologist John Sweller, who believes that cognitive load is the amount of activity that is applied to a person's cognitive system when a person completes an activity at a given time[2]. According to the relevant research, cognitive load is the level of psychological tolerance shown by processing a specific amount of information[3]. As the amount of information received and processed by individuals increases, the cognitive load increases gradually[4]. It is not difficult to find that cognitive load can be understood as an individual receiving more information at the same time than the maximum amount received by the brain, resulting in information redundancy[5]. In the digital interface, the main performance is the same interface information is too large, the user can not filter out useful interface information in a short period of time, resulting in brain information redundancy, thus forming cognitive load.

\subsection{User Emotional Experience and Availability Testing}

The user experience is user-centric, emphasizing that the product is for the user, not just to implement certain features[6]. In general, the user experience is to understand the user's needs, motivations, and emotions and product interactions in a particular scenario [7]. Mainly focus on the availability of products, functional ease of use, availability is mainly to ensure that users can smoothly complete the basic needs of users when using the product. Ease of use is mainly reflected in the product can exceed the user's psychological expectations, so that users meet the excitement needs, The ease of use is mainly reflected in the ability of the product to exceed the user's psychological expectations and the user's ability to meet the excitement needs[8]. Therefore, the availability and ease of use of community mobile healthcare systems play a critical role in the user experience. In this study, System Usability Scale is mainly used for usability and ease of use research.

\footnotetext{
*Corresponding author’s e-mail: 544253480@qq.com.
} 
In addition, psychological studies have shown that cognitive models do not fully include behavioral factors, and that emotional experience plays an important role[9]. It not only influences the beliefs and attitudes of the individual, but also guides the individual in decisionmaking and action[10]. Compared with data analysis research, the user's emotional experience can reflect the user's real needs, there are studies Emotional changes such as joy, frustration, and joy can capture the experience of users using information products[10-11]. Therefore, in the user's emotional experience, Positive and Negative Affect Scale is used to study the user's emotional experience, including psychological efforts and task difficulty evaluation[12].

\section{Experimental scheme design}

\subsection{Experimental design}

This experiment mainly takes "Wanjia Medical APP" and "Spring Rain Medical APP" as the test software, using 2 (cognitive load: high, low) x 5 (task type: login account, click on patient appointment, fill out patient information, fill out prescription, Charge for prescription) multi-factor mixed experimental design, in which cognitive load is the inter-test factor; the task type is the subject factor, the variables include a cognitive negative index, time to complete a task, learning ability, etc. This experiment requires the collection of two sets of data samples: objective data and subjective data. Objective data refer to behavioral data during the experiment. Subjective data is included in PANAS and SUS data.This experiment mainly takes "Wanjia Medical APP" and "Spring Rain Medical APP" as the test software, using 2 (cognitive load: high, low) x 5 (task type: login account, click on patient appointment, fill out patient information, fill out prescription, Charge for prescription) multi-factor mixed experiment design, in which cognitive load is the inter-test factor, the task type is the subject factor, the variables include cognitive negative index, time to complete a task, learning ability, etc. This experiment requires the collection of two sets of data samples: objective data and subjective data. Objective data refer to behavioral data during the experiment. Subjective data are included in PANAS and SUS data.
In this study, 20 community doctors who had not used the app were randomly selected to participate in the experiment, with an average age of $43.6 \pm 1.05$ years of age, education in high school or above, community medical work for more than 10 years, including 12 men, women 8 people. All subjects were fluent in the use of APP software, vision or corrective vision is normal, are right hand, and are voluntary to participate in the experiment. The subjects were tested in the same environment, using iPhone $\mathrm{X}$ phones and using digital cameras to record the subjects and record the time the task completes, while rendering the control of the time.

\subsection{Experimental procedures}

According to the mixed experimental design scheme, the experiment divided 20 subjects into 2 groups of 10 people per group (one group for high cognitive load, one group for low cognitive load), each subject participated in the above two APP task process tests. Before the experiment, subjects were asked to listen to the requirements of the experiment, according to the requirements to fill in personal information and pre-test PANAS, PANAS using a 5-level scoring system for scoring, consisting of 10 positive emotional words and 10 negative emotional words[13].Ask subjects to rate the vocabulary in the scale according to their own operating experience, 1 is very relaxed and very easy, 5 is very hard and very difficult.During the experiment, the subjects completed the tasks in order as required, and the experimenters recorded the time required for each task and the time required for the entire task process.After each participant completes the task process, fill out PANAS and SUS after testing. After a ten-minute break, conduct a second mission flow experiment.

\section{Experimental data analysis}

\subsection{Behavioral data analysis}

At the end of the experiment, SPSS statistical analysis software is used to describe statistics, anovaise and related analysis of each task. And cognitive load analysis of each group of subjects.the results of the analysis as shown in Table 1.

\subsection{Experimental test subjects and instruments}

Table 1. Descriptive results of cognitive load measurement in each group

\begin{tabular}{|c|c|c|c|c|c|c|c|c|}
\hline Cognitive load & Tas & & MAX & MIN & $\mathbf{M}$ & SD & $\mathbf{P}$ & $\mathbf{N}$ \\
\hline \multirow{7}{*}{$\begin{array}{l}\text { Low cognitive } \\
\text { load }\end{array}$} & \multirow{5}{*}{$\begin{array}{l}\text { wanjia } \\
\text { medical } \\
\text { APP }\end{array}$} & Step 1 & $9.6 \mathrm{~s}$ & $7.3 \mathrm{~s}$ & $8.3 \mathrm{~s}$ & $1.1 \mathrm{~s}$ & 0.43 & 10 \\
\hline & & Step 2 & $5.3 \mathrm{~s}$ & $2.8 \mathrm{~s}$ & $3.6 \mathrm{~s}$ & $1.2 \mathrm{~s}$ & 0.36 & 10 \\
\hline & & Step 3 & $61.6 \mathrm{~s}$ & $40.3 \mathrm{~s}$ & $45.6 \mathrm{~s}$ & $1.3 \mathrm{~s}$ & 0.01 & 10 \\
\hline & & Step 4 & $72.4 \mathrm{~s}$ & $52.1 \mathrm{~s}$ & $65.6 \mathrm{~s}$ & $1.6 \mathrm{~s}$ & 0.07 & 10 \\
\hline & & Step 5 & $15.7 \mathrm{~s}$ & $10.4 \mathrm{~s}$ & $12.6 \mathrm{~s}$ & $1.2 \mathrm{~s}$ & 0.39 & 10 \\
\hline & \multirow{2}{*}{$\begin{array}{l}\text { chunyumed } \\
\text { ical APP }\end{array}$} & Step 1 & $10.4 \mathrm{~s}$ & $6.9 \mathrm{~s}$ & $8.1 \mathrm{~s}$ & $1.2 \mathrm{~s}$ & 0.52 & 10 \\
\hline & & $\mathrm{S}$ tep 2 & $5.0 \mathrm{~s}$ & $2.4 \mathrm{~s}$ & $3.2 \mathrm{~s}$ & $1.1 \mathrm{~s}$ & 0.38 & 10 \\
\hline
\end{tabular}




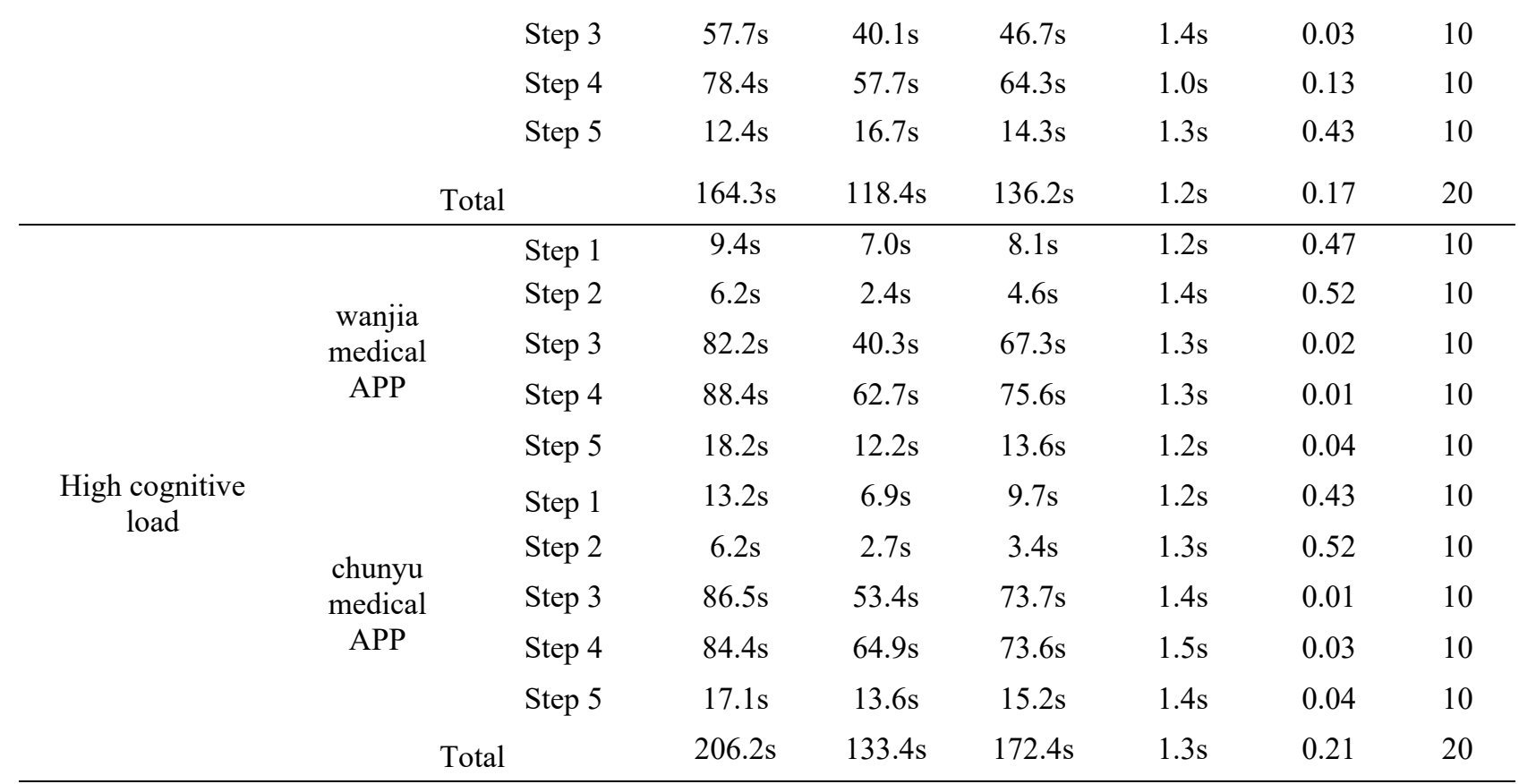

The experimental results show that the low cognitive load is faster than that of a person with a high cognitive load. At the same time, the main effect of the task category is significant. After simple tests of high and low cognitive load effects, there were significant differences in the "filling in patient information", "filling out prescriptions" and "paying prescriptions" in the task category $(\mathrm{P}<0.05)$ 。 At the same time, the tasks with significant differences in cognitive load in the task category were compared, and it was found that the completion time of the low cognitive load group $(136.2 \pm 1.2)$ was lower than that of the high-altitude cognitive load group $(172.4 \pm 1.3)$ 。 Overall, the cognitive load of the user was significantly different in the cognitive load regardless of the user's low or high cognitive load, the significant difference in the cognitive load group was greater in the high cognitive load group, and the cognitive load of wanjia medical APP $\{(143.8$ $\left.\pm 1.2)_{\text {low }} ;(169.2 \pm 1.3)_{\text {high }}\right\}$ was slightly higher than that of chunyu medical APP $\left\{(137.6 \pm 1.2)_{\text {low }} ;(175.6 \pm 1.4)_{\text {high }}\right\}$.

\subsection{Emotional experience and usability analysis}

The measurement of emotional experience was measured with a 5-level Positive and Negative Affect Scale, before the experiment, the subjects filled out the first scale, respectively, to obtain the pre-measurement values of positive emotion (PA1) and negative emotion (NA1), and after the test was completed, the test subjects were asked to fill out the positive emotion (PA2) and negative emotion (NA2) after the measurement. And calculate the change value of positive and emotional experience: positive emotion (PA2) - Positive Emotion (PA1), and Negative Emotional Experience Change Value: Negative Emotion (NA2) - Negative Emotion (NA2). The description statistics for emotional experience measurements are as shown in Table 2.

Table 2. Test the task flow

\begin{tabular}{cccccccc}
\hline condition & statistical type & PA1 $_{1}$ & NA1 $_{1}$ & PA2 $_{2}$ & NA2 $_{2}$ & PA & NA \\
\hline high cognitive load & Mean & 3.27 & 1.13 & 3.73 & 1.47 & -0.42 & 0.21 \\
Wanjia APP & SD & 0.52 & 0.67 & 0.22 & 0.31 & 0.32 & 0.26 \\
high cognitive load & Mean & 3.27 & 1.13 & 3.81 & 1.08 & 0.45 & -0.08 \\
Chunyu APP & SD & 0.52 & 0.67 & 0.30 & 0.35 & 0.37 & 0.41 \\
low cognitive load & Mean & 3.27 & 1.13 & 3.36 & 1.62 & 0.26 & -0.18 \\
Wanjia APP & SD & 0.52 & 0.67 & 0.54 & 0.37 & 0.32 & 0.37 \\
low cognitive load & Mean & 3.27 & 1.13 & 3.42 & 1.84 & 0.33 & -0.12 \\
Chunyu APP & SD & 0.52 & 0.67 & 0.43 & 0.18 & 0.07 & 0.21 \\
\hline
\end{tabular}

From the comparison of the mean measurement of the emotional experience of the subjects, it was found that the chunyu APP under high cognitive load reduced the average positive emotional experience value of the subjects $(\mathrm{PA}=-0.42)$, while the chunyu APP decreased the negative emotional experience value $(\mathrm{NA}=$ -
0.08).Low cognitive load chunyu APP and wanjia APP increased the positive emotional experience of the subjects, and negative emotional experience decreased.Overall, positive emotional experience under low cognitive load was higher than high cognitive load, 
and the negative and negative emotional experience under low cognitive load had less effect.

After completing the emotional experience experiment, the researchers were asked to fill out the SUS availability scale to measure the user's analysis of app availability in the experiment. According to the SUS test specification, the scale questionnaire totals 10 questions and uses a five-level likert scale(0-4), such as Table 3.

Table 3. Analysis of user availability and ease of use

\begin{tabular}{|c|c|c|c|c|c|}
\hline subject & $\begin{array}{l}\text { raw } \\
\text { score }\end{array}$ & $\begin{array}{l}\text { conversion } \\
\text { score }\end{array}$ & $\begin{array}{l}\text { SUS } \\
\text { score }\end{array}$ & $\begin{array}{c}\text { SUS Easy } \\
\text { Learning Index }\end{array}$ & $\begin{array}{c}\text { SUS } \\
\text { Availability } \\
\text { Index } \\
\end{array}$ \\
\hline I think I'll use the system a lot & 4.6 & 3.6 & \multirow{10}{*}{$\begin{array}{l}51 \\
(\mathrm{~F})\end{array}$} & \multirow{10}{*}{$\begin{array}{l}36.3 \\
(\mathrm{~F})\end{array}$} & \multirow{10}{*}{$\begin{array}{l}52.2 \\
(\mathrm{D})\end{array}$} \\
\hline I think the system is more complicated & 4.0 & 1.0 & & & \\
\hline I think the system is simple and easy to use & 3.1 & 2.1 & & & \\
\hline I think professional guidance is needed & 3.1 & 1.9 & & & \\
\hline I think the system is better connected togethe & 3.2 & 2.2 & & & \\
\hline I think there's a lack of continuity in the system & 3.3 & 1.7 & & & \\
\hline I think most people learn quickly & 2.8 & 1.8 & & & \\
\hline I find the system difficult to use & 3.2 & 1.8 & & & \\
\hline I feel confident when I use the system & 3.1 & 2.1 & & & \\
\hline I need to learn a lot to use & 2.8 & 2.2 & & & \\
\hline
\end{tabular}

Through the SUS availability scale filled out by the subjects, it is known that the user's SUS score for the APP in the experiment is 51, and the query SUS database shows that less than $14 \%$ of the users are satisfied with the APP in the experiment. In terms of ease of learning, user satisfaction was also less than $14 \%$, and usability was slightly higher, about $15 \%-34 \%$. From the experimental data, it can be found that the subjects are available for the APP ease of use in the experiment low sexual satisfaction.

\section{$5 \quad$ Analysis and Summary}

Firstly,through experiments found that the participants' cognitive patterns are generally: visual perception interface-brain cognition-start operation-information feedback, when the subject sencountered an operational error or can not find the function, often appear suspicious, anxious behavior, and will be based on their own cognition to start to try some operations. Therefore, it can be seen that the frustration caused by cognitive error can affect the user's operation and reduce the performance of the system.

Secondly, from the APP test data, it can be seen that high cognitive load has a significant effect on completing tasks three, four and five, it can be inferred that cognitive load has a certain effect on the time and efficiency of completing the task.

Finally, in the analysis of emotional experience and availability, it is found that high cognitive load has a certain influence on the positive and negative emotional experience of users, and the two diagnostic APP in the experiment is low on ease of use and usability satisfaction, so it can be inferred that the product availability, ease of use of the deficiency, but also on the user's emotional experience has a certain impact.
Therefore, through the above experiments, it can be concluded that the overall use of the two applications in the test is good, but the "fill in patient information" and "fill in prescription" functions need to be further optimized to improve the user experience.

\section{Acknowledgments}

Fund Project: Special topic of art education in Shandong Province "Application and Exploration of Cognitive Load Theory in the Practice of Digital Media Art Education" (Project No.YJ20191085)

\section{References}

1. Li Fangyu, Ni Jia. Research on the Applicability of Old-age User 's Mental Model in Smart Kitchens 'Dietary Management [J] .Journal of Graphics, 2018,39(04): 689-694.

2. Sweller J. Cognitive load during problem solving,effects on learning [J].Cognitive Science, 1988,12(2):257-285.

3. Cooper,G. Cognitive load theory as an aid for instructional design[J]. Australia Journal of Educational Technology,1990,6(1):108-113.

4. Xin Ziqiang, Lin Chongde. The relationship between cognitive load and cognitive skills and schema acquisition and its teaching significance [J]. Journal of East China Normal University (EDUCATION SCIENCE EDITION), 2002,2(4): 55-60.

5. Paas,F.,\&Van Merrienboer,J.J.G.Instructional control of cognitive load in the training ofcomplex cognitive tasks.[J].Educational Psychology Review, 1994,6(4):351-371. 
6. You Fang, Wang Jianmin, Xiao Jingru. Interactive design thinking and practice [M]. Electronic Industry Press, 2017.

7. Hassenzahlm,Tractinskyn.User experience- a researchagenda[J].Behavior\& technology,2006, 25(2):91-97.

8. Lan Yuqi, Liu Pai. Research on interactive product Emotionalization based on user experience [J]. Packaging engineering, 2019,40 (12): 23-28

9. Hou Guanhua, Dong Hua, Liu Ying, fan Guangrui. An Empirical Study on the impact of navigation structure and cognitive load on the user experience of the elderly facing the Digital Library -- Taking the National Digital Library as an example [J]. Library and information work, 2018, 62 (13): 48-53.

10. Ping Z.A theoretical framework of affective concepts and their relationships in the ICT Context[J].MIS Quarterly,2013,37(1):247-74.

11. Liu Luchuan, Sun Kai. Relationship between emotional experience and satisfaction of social media users: a case study of microblog [J]. Journal of China Library, 2015,41 (01): 76-91.

12. Sun Chongyong, Li Shulian. Cognitive load theory and its application in teaching design [M]. Tsinghua University Press, 2017. 